\title{
SOME PROPERTIES OF THE POLYNOMIALLY BOUNDED O-MINIMAL EXPANSIONS OF THE REAL FIELD AND OF SOME QUASIANALYTIC LOCAL RINGS
}

\author{
BERRAHO M.
}

\begin{abstract}
In this paper, we study the Weierstrass division theorem over the rings of smooth germs that are definable in an arbitrary polynomially bounded o-minimal expansion of the real field by giving some criteria for satisfying this theorem. Afterwards, we study some topological properties of some quasianalytic subrings of the ring of smooth germs for the $\left(x_{1}\right)$-adic topology by showing that these rings are separable metric spaces. Also, we cite a criterion for their completeness with respect to the $\left(x_{1}\right)$-adic topology.

Key words and phrases: Weierstrass division theorem, polynomially bounded o-minimal structure, quasianalytic ring, $\left(x_{1}\right)$-adic topology.
\end{abstract}

Faculty of Sciences, Ibn Tofail University, PO Box 242, Kenitra, Morocco

E-mail: b.mourad87@hotmail.com

\section{INTRODUCTION}

In this paper, we will study the Weierstrass division theorem over some polynomially bounded o-minimal structures in order to tackle a question asked by L. Van den Dries in [18]: does the Weierstrass division theorem hold over the ring of real analytic germs that are definable in an o-minimal structure (not necessarily polynomially bounded), extending the structure of real numbers? In [9], there is a positive answer to this question for the semialgebraic setting and also for the structure of globally subanalytic sets and functions, but, a negative answer for the structure $(\mathbb{R},+,-, ., 0,1,<, \exp )$. The Weierstrass division theorem is the key tool for local complex analytic geometry (see, for example, [8, Chapter II and III]). It is also used e.g. in the proof of the important Oka's coherence theorem (see [8, Chapter IV]). So, V. Thelliez have shown in [17] that over the ring of germs of smooth functions in some fixed quasianalytic Carleman class that is closed under derivation, this theorem holds only for hyperbolic polymomials (see also [6]). In [7], it has been shown that for the ring of smooth germs that are definable in a polynomially bounded o-minimal expansion of the real field, this theorem holds just for the hyperbolic polymomials. Also in [14], it has been shown in particular that the Weierstrass division theorem does not hold for the ring of the smooth germs that are definable in a polynomially bounded o-minimal expansion of the real field that contains strictly the ring of real analytic germs. So, in the third section, we try to generalize the result given in [7] by studying the Weierstrass division theorem over a polynomially bounded o-minimal expansion of the real field for arbitrary functions that are regular of an order $p$ with respect to the last 
variable, so we give a negative answer to this problem and thanks to the result proved in [13], we will form some criterions for satisfying this theorem.

In commutative algebra, the filtration on a commutative ring $R$ by the powers of a proper ideal $I$ determines the Krull topology (after Wolfgang Krull) or the $I$-adic topology on $R$. The case of a maximal ideal $I=m$ is especially important, for example the distinguished maximal ideal of a valuation ring. The basis of open neighbourhoods of 0 in $R$ is given by the powers $\left(I^{n}\right)_{n \in \mathbb{N}}$. In the last section, we are going to study some $\left(x_{1}\right)$-adic topological properties over some quasianalytic subrings of the ring of smooth germs, we endow these rings with a metric which turns them into a separable metric spaces, also we study their completeness for the $\left(x_{1}\right)$-adic topology.

\section{NOTATIONS AND DEFINITIONS}

Thoughout this paper, $\mathcal{R}$ denotes a fixed (but arbitrary) expansion of the structure $\overline{\mathbb{R}}=$ $(\mathbb{R},<, 0,1,+,-,$.$) . Definable means first order definable in \mathcal{R}$ with parameters from $\mathbb{R}$. A function $f: X \rightarrow \mathbb{R}$ is said to be definable if its graph is definable. We say that $\mathcal{R}$ is o-minimal if every definable subset of $\mathbb{R}$ is just a finite union of intervals and points. We say that $\mathcal{R}$ is polynomially bounded if for every definable function $f: \mathbb{R} \rightarrow \mathbb{R}$ there exists $N \in \mathbb{N}$ such that $|f(t)| \leq t^{N}$ for all sufficiently large positive $t$.

Example 1. The structure $\overline{\mathbb{R}}:=(\mathbb{R},+,-, ., 0,1,<)$ is polynomially bounded and o-minimal (by Tarski-Seidenberg); the sets definable in this structure are exactly the semialgebraic sets.

The structure of the ordered real field with restricted analytic functions $\mathbb{R}_{a n}$, whose definable sets are the finitely subanalytic sets, is a polynomially bounded o-minimal structure by [10].

For each $n \in \mathbb{N}$, we denote by $\mathcal{E}_{n}$ the ring of smooth germs at the origin of $\mathbb{R}^{n}$ and by $\mathbb{R}\left[\left[x_{1}, \ldots, x_{n}\right]\right]$ the ring of formal power series with coefficients in $\mathbb{R}$.

For every $f \in \mathcal{E}_{n}$, we denote by $\hat{f} \in \mathbb{R}\left[\left[x_{1}, \ldots, x_{n}\right]\right]$ its (infinite) Taylor expansion at the origin. The mapping $\mathcal{E}_{n} \ni f \mapsto \hat{f} \in \mathbb{R}\left[\left[x_{1}, \ldots, x_{n}\right]\right]$ is called the Borel mapping.

Let $\hat{f}=\sum_{n \in \mathbb{N}} a_{n} x_{1}{ }^{n} \in \mathbb{R}\left[\left[x_{1}\right]\right]$ be a formal power series. We denote by Supp $\hat{f}=\{n \in \mathbb{N}$ : $\left.a_{n} \neq 0\right\}$.

Definition 1. For each $n \in \mathbb{N}$, let $\mathcal{C}_{n} \subset \mathcal{E}_{n}$ be a subring of the ring of germs of smooth functions at the origin of $\mathbb{R}^{n}$. We say that $\mathcal{C}_{n}$ is a quasianalytic ring if the Borel mapping ${ }^{\wedge}: \mathcal{C}_{n} \rightarrow$ $\mathbb{R}\left[\left[x_{1}, \ldots, x_{n}\right]\right]$ is injective. In other words, if the ring $\mathcal{C}_{n}$ does not contain any nonzero smooth flat germ at 0 .

Example 2. a) The rings $\mathcal{A}_{n}, n \in \mathbb{N}$, of germs of the real analytic functions at $0 \in \mathbb{R}^{n}$ are quasianalytic rings.

b) The ring of smooth germs $\mathcal{E}_{1}$ is not a quasianalytic ring as it contains some nonzero smooth germs that vanish at 0 together with all their derivatives.

By quasianaliticity, we have that $\hat{\mathcal{C}_{n}} \subset \mathbb{R}\left[\left[x_{1}, \ldots, x_{n}\right]\right]$ for each $n \in \mathbb{N}$.

A germ $f \in \mathcal{C}_{n}$ is called regular in $x_{n}$ of order $p$ with respect to the variable $x_{n}$ if there exists a formal power series $h \in \mathbb{R}\left[\left[x_{1}, \ldots, x_{n}\right]\right]$ such that $\hat{f}=x_{n}^{p} h\left(0, x_{n}\right)$ with $h(0) \neq 0$. 
Let $\left\{\mathcal{C}_{n}: n \in \mathbb{N}\right\}$ be a system of quasianalytic rings, we say that this system satisfies Weierstrass division theorem if $f, g \in \mathcal{C}_{n}$ such that the germ $g$ is regular of order $p$ with respect to the variable $x_{n}$, there exist $q \in \mathcal{C}_{n}$ and $r_{1}, \ldots, r_{p} \in \mathcal{C}_{n-1}$ such that

$$
f=g q+\sum_{j=1}^{p} r_{j}\left(x_{1}, \ldots, x_{n-1}\right) x_{n}^{p-j} .
$$

Example 3. The system of the rings of real analytic germs at the origin $\left\{\mathcal{A}_{n}: n \in \mathbb{N}\right\}$ satisfies the Weierstrass division theorem.

\section{WEIERSTRASS DIVISION THEOREM OVER SOME POLYNOMIALLY BOUNDED O-MINIMAL STRUCTURES}

Fix a polynomially bounded o-minimal structure $\mathcal{R}$ that is an expansion of the structure $\overline{\mathbb{R}}=(\mathbb{R},<, 0,1,+,-,$.$) and denote by \left(\mathcal{D}_{n}\right)_{n \in \mathbb{N}}$ the rings of those smooth functions germs at 0 which are definable in $\mathcal{R}$. We recall that if $\mathcal{R}=(\mathbb{R},<, 0,1,+,-,$.$) , then the rings \left(\mathcal{D}_{n}\right)_{n \in \mathbb{N}}$ are exactly the rings of algebraic smooth functions germs called Nash germs (see [5] for a thorough treatment of Nash germs).

It is well known thanks to [11], that the rings $\left(\mathcal{D}_{n}\right)_{n \in \mathbb{N}}$ are also a famous example of quasianalytic local rings.

We recall that in every polynomially bounded o-minimal structure $\mathcal{R}$, the family $\left(\mathcal{D}_{n}\right)_{n \in \mathbb{N}}$ satisfies the following assertions:

(C1) the rings $\left(\mathcal{D}_{n}\right)_{n \in \mathbb{N}}$ are closed under monomial division, so each $\mathcal{D}_{1}$ is a principal ideal domain;

(C2) the closedness under inverse implies that each $\mathcal{D}_{n}$ is a local ring. The unique maximal ideal of each ring $\mathcal{D}_{n}$ is generated by the coordinates $x_{1}, \ldots, x_{n}$;

(C3) the rings $\left(\mathcal{D}_{n}\right)_{n \in \mathbb{N}}$ are closed under partial differentiation.

We know thanks to [5, theorem 8.2.2] that the ring of smooth germs that are definable in the structure $\overline{\mathbb{R}}$ (called Nash germs) satisfies the Weierstrass division theorem, but unfortunately, there are some rings $\mathcal{D}_{n}$ which don't satisfy the Weierstrass division theorem even if we take them to be the rings of real analytic functions that are definable in a polynomially bounded o-minimal structure $\mathcal{R}$, which is the aim of the following proposition.

We recall that the o-minimal structure $\mathcal{R}=(\mathbb{R}, \sin \lceil[0,1])$ (where $\sin \lceil[0,1]$ denotes the restriction of the sin function to the interval $[0,1])$ is polynomially bounded as a reduct (in the sense of definability) of the polynomially bounded o-minimal structure $\mathbb{R}_{a n}$ (the definable sets in $\mathbb{R}_{a n}$ are exactly the finitely subanalytic sets).

Remark 1. By $\left[3\right.$, Proposition 3.1], if the system $\left(\mathcal{D}_{n}\right)_{n \in \mathbb{N}}$ satisfies the Weierstrass division theorem, then so does the system of the rings of real analytic definable germs in such structures. But the reciprocal does not hold true by [3, Remark 3.3].

Proposition 1. Let $\mathcal{R}=(\overline{\mathbb{R}}, \sin \lceil[0,1])$, then the Weierstrass division theorem does not hold in the ring $\mathcal{D}_{2}$. 
Proof. Suppose that the Weierstrass division theorem holds in $\mathcal{D}_{2}$, the function cos is also definable in this structure $\mathcal{R}$ as it is closed under partial differentiation. So, we deduce the existence of $Q_{1}, Q_{2}$ in $\mathcal{D}_{2}$, and $R_{i}, T_{i}$ in $\mathcal{D}_{1}, i=0,1$, such that:

$$
\begin{aligned}
& \sin (y)=\left(x^{2}+y^{2}\right) Q_{1}(x, y)+R_{1}(x) y+R_{0}(x), \\
& \cos (y)=\left(x^{2}+y^{2}\right) Q_{2}(x, y)+T_{1}(x) y+T_{0}(x) .
\end{aligned}
$$

It is well known that $\exp (x)=\cos (i x)-i \sin (i x)$ for all $x \in \mathbb{R}$. By taking $y=i x$ in (1) and (2), we have that

$$
\exp (x)=T_{0}+i x T_{1}-i\left(R_{0}+i x R_{1}\right)=\left(T_{0}+x R_{1}\right)+i\left(x T_{1}-R_{0}\right) .
$$

So, $\exp =T_{0}+x R_{1}$.

Therefore, the restriction of the function exp to the interval $[0,1]$ is definable in the structure $\mathcal{R}=(\overline{\mathbb{R}}, \sin \lceil[0,1])$, which contradicts Bianconi's theorem in [4]. Hence, the Weierstrass division theorem does not hold in the ring $\mathcal{D}_{2}$.

So, under what conditions this system of the rings $\left(\mathcal{D}_{n}\right)_{n \in \mathbb{N}}$ satisfies the Weierstrass division theorem? That is why, we will give some criteria for satisfying this theorem.

For each $n \in \mathbb{N}$, let $f, g \in \mathcal{D}_{n}$ be such that $g$ is regular of order $p$ with respect to $x_{n}$, by the Weierstrass division theorem in the ring of formal power series $\mathbb{R}\left[\left[x_{1}, \ldots, x_{n}\right]\right]$, there exist $\tilde{q} \in \mathbb{R}\left[\left[x_{1}, \ldots, x_{n}\right]\right], \tilde{r}_{1}, \ldots, \tilde{r}_{p} \in \mathbb{R}\left[\left[x_{1}, \ldots x_{n-1}\right]\right]$ such that

$$
\hat{f}=\hat{g} \tilde{q}+\sum_{j=1}^{p} \tilde{r}_{j}\left(x_{1}, \ldots, x_{n-1}\right) x_{n}^{p-j} .
$$

Put $\tilde{R}=\sum_{j=1}^{p} \tilde{r}_{j}\left(x_{1}, \ldots, x_{n-1}\right) x_{n}^{p-j}$.

Theorem 1. For each $n \in \mathbb{N}$, let $f$ and $g$ be elements of the ring $\mathcal{D}_{n}$ and the division as in the equation (3), so, if there exists $R \in \mathcal{D}_{n}$ such that $\hat{R}=\tilde{R}$, then this division holds in the ring $\mathcal{D}_{n}$.

Proof. We have that $\tilde{R}=\sum_{j=1}^{p} \tilde{r}_{j}\left(x_{1}, \ldots, x_{n-1}\right) x_{n}^{p-j}$, hence we have

$$
\frac{\partial^{p-1} \tilde{R}}{\partial x_{n}^{p-1}}=(p-1) ! \tilde{r}_{1}\left(x_{1}, \ldots, x_{n-1}\right) .
$$

But we have

$$
\left(\frac{\partial^{p-1} R}{\partial x_{n}^{p-1}}\right)=\frac{\partial^{p-1} \tilde{R}}{\partial x_{n}^{p-1}}=(p-1) ! \tilde{r}_{1}\left(x_{1}, \ldots, x_{n-1}\right) .
$$

As the rings $\left(\mathcal{D}_{n}\right)_{n \in \mathbb{N}}$ are closed under partial differentiation, we deduce that there exists $r_{1}$ such that $\hat{r_{1}}=\tilde{r}_{1}$.

We have $\frac{\partial^{p-2} R}{\partial x_{n}^{p-2}}=(p-1) ! \tilde{r}_{1}\left(x_{1}, \ldots, x_{n-1}\right) x_{n}+(p-2) ! \tilde{r}_{2}\left(x_{1}, \ldots, x_{n-1}\right)$, for the same reason we deduce that there exists $r_{2} \in \mathcal{D}_{n-1}$ such that $\hat{r_{2}}=\tilde{r_{2}}$. By continuing this process, we prove that there exist

$$
r_{1}, \ldots, r_{p} \in \mathcal{D}_{n-1} \quad \text { such that } \quad \hat{r}_{j}=\tilde{r}_{j}, \forall j=1, \ldots, p .
$$


By (3), we have

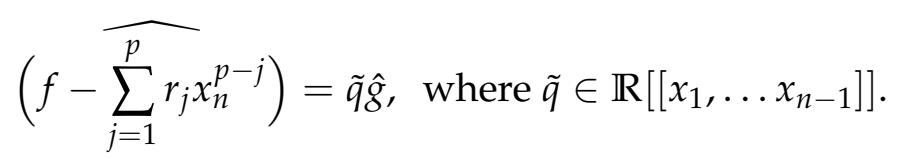

By a result of Krzysztof Jan Nowak [13], Criterion of Divisibility, there exists $q \in \mathcal{D}_{n}$ such that

$$
f-\sum_{j=1}^{p} r_{j} x_{n}^{p-j}=q g
$$

which proves the theorem.

We know that the quasianalytic rings $\left(\mathcal{D}_{n}\right)_{n \in \mathbb{N}}$ contain the polynomial ring and are closed under composition and partial differentiation. So, they satisfy the assertions of Definition 1.1 in [16], by [16, Corollary 2.4], the Borel mapping $\wedge: \mathcal{D}_{n} \rightarrow \mathbb{R}\left[\left[x_{1}, \ldots, x_{n}\right]\right]$ is not surjective if $n \geq 2$, that is why we must restrict the following two propositions just to the case when $n=1$.

Recall that a polynomial $a_{k} x_{2}^{k}+a_{k-1} x_{2}^{k-1}+\ldots+a_{0} \in \mathbb{R}\left[\left[x_{1}\right]\right]\left[x_{2}\right]$ is a distinguished polynomial if $a_{k}=1$ and $a_{0}(0)=\ldots=a_{k-1}(0)=0$.

Proposition 2. If the Borel mapping ${ }^{\wedge}: \mathcal{D}_{1} \rightarrow \mathbb{R}\left[\left[x_{1}\right]\right]$ is surjective, then the Weierstrass preparation theorem holds in the ring $\mathcal{D}_{2}$.

Proof. Let $f \in \mathcal{D}_{2}$ such that $\hat{f}$ is regular of order $p$ with respect to $x_{2}$, so, by the Weierstrass preparation theorem in the ring of formal power series $\mathbb{R}\left[\left[x_{1}, x_{2}\right]\right]$, there exists a distinguished polynomial $P \in \mathbb{R}\left[\left[x_{1}\right]\right]\left[x_{2}\right]$ of degree $p$ with respect to $x_{2}$, such that $\hat{f}=g P$, where $g \in \mathbb{R}\left[\left[x_{1}, x_{2}\right]\right]$, and $g(0) \neq 0$, and $P=x_{2}^{p}+\tilde{a}_{p-1}\left(x_{1}\right) x_{2}^{p-1}+\ldots+\tilde{a_{0}}\left(x_{1}\right)$.

As the Borel mapping is surjective, there exist $a_{0}, \ldots, a_{p-1} \in \mathcal{D}_{1}$ such that

$$
\hat{a}_{j}=\tilde{a}_{j} \text { with } a_{j}(0)=0, \quad \forall j=0, \ldots, p-1 .
$$

Put $P_{f}=x_{2}^{p}+a_{p-1}\left(x_{1}\right) x_{2}^{p-1}+\ldots+a_{0}\left(x_{1}\right)$, so, $\hat{f}=g \hat{P}_{f}$.

By the result of Krzysztof Jan Nowak in [13], Criterion of Divisibility, there exists $q \in \mathcal{D}_{2}$ such that $f=q P_{f}$, as $\hat{q}=g$ we deduce that $q(0) \neq 0$, which proves the proposition.

Proposition 3. If the Borel mapping ${ }^{\wedge}: \mathcal{D}_{1} \rightarrow \mathbb{R}\left[\left[x_{1}\right]\right]$ is surjective, then the Weierstrass division theorem holds in the ring $\mathcal{D}_{2}$.

Proof. Let $f, g \in \mathcal{D}_{2}$ be such that $\hat{g}$ is regular of order $p$ with respect to $x_{2}$, by the Weierstrass division theorem in the ring of formal power series $\mathbb{R}\left[\left[x_{1}, x_{2}\right]\right]$, there exist $\tilde{q} \in \mathbb{R}\left[\left[x_{1}, x_{2}\right]\right]$, $\tilde{r}_{1}, \ldots, \tilde{r}_{p} \in \mathbb{R}\left[\left[x_{1}\right]\right]$ such that

$$
\hat{f}=\hat{g} \tilde{q}+\sum_{j=1}^{p} \tilde{r}_{j}\left(x_{1}\right) x_{2}^{p-j} .
$$

By assumption, the Borel mapping is surjective, so there exist $r_{1}, \ldots, r_{p} \in \mathcal{D}_{1}$ such that

$$
\hat{r}_{j}=\tilde{r_{j}}, \quad \forall j=1, \ldots, p,
$$

so (4) implies that

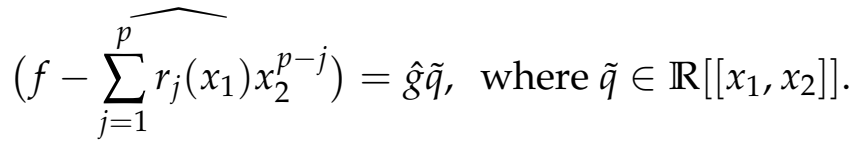


By the result of Krzysztof Jan Nowak in [13], Criterion of Divisibility, there exists $q \in \mathcal{D}_{2}$ such that

$$
f-\sum_{j=1}^{p} r_{j}\left(x_{1}\right) x_{2}^{p-j}=g q,
$$

which proves the proposition.

Remark 2. Proposition 2 is an immediate consequence of Proposition 3, therefore, it suffices to prove Proposition 3 to obtain the Proposition 2.

Remark 3. By imitating the classical proof of the noetherianity of the ring of convergent power series as a consequence of the satisfaction of the Weierstrass division theorem, we deduce that if the Borel mapping ${ }^{\wedge}: \mathcal{D}_{1} \rightarrow \mathbb{R}\left[\left[x_{1}\right]\right]$ is surjective, then the ring $\mathcal{D}_{2}$ is noetherian.

We end this section by another application of the result of Krzysztof Jan Nowak. For this aim, let us recall the following theorem proved in [15, Theorem 10.1].

$$
\begin{gathered}
\text { Put } f=\sum_{n=0}^{+\infty} f_{n} x_{1}^{n} \in \mathbb{R}\left[\left[x_{1}\right]\right], \text { let } g_{1}, \ldots, g_{s} \text { be elements of } \mathbb{R}\left[\left[x_{1}\right]\right] . \text { Set } \\
\qquad e(f):=\min \left\{n \in \mathbb{N}: f_{n} \neq 0\right\}=\inf \operatorname{Supp}(f), \\
\Delta_{1}=e\left(g_{1}\right)+\mathbb{N},
\end{gathered}
$$

and

$$
\Delta_{i}=e\left(g_{i}\right)+\mathbb{N} \backslash \bigcup_{1 \leq j<i} \Delta_{j} \text { for all } 2 \leq i \leq s .
$$

Finally, set $\Delta_{0}:=\mathbb{N} \backslash \bigcup_{1 \leq i \leq s} \Delta_{i}$.

Theorem 2. Let $f \in \mathbb{R}\left[\left[x_{1}\right]\right]$. Then there exist some unique power series $q_{1}, \ldots, q_{s}, r \in \mathbb{R}\left[\left[x_{1}\right]\right]$ such that

$$
f=g_{1} q_{1}+\ldots+g_{s} q_{s}+r
$$

and

$$
e\left(g_{i}\right)+\operatorname{Supp}\left(q_{i}\right) \subset \Delta_{i} \text {, and } \operatorname{Supp}(r) \subset \Delta_{0} .
$$

The power series $r$ is called the remainder of the division of $f$ by $g_{1}, \ldots, g_{s}$.

Proposition 4. Let I be an ideal of the ring $\mathcal{D}_{1}$, then every element of this ring is equivalent modulo I to a polynomial.

Proof. Let $I$ be a nonzero ideal of the ring $\mathcal{D}_{1}$, where $\mathcal{D}_{1}$ is a principal ideal domain and this ideal $I$ is generated by a single element $g$. Let $f$ be an arbitrary element of the ring $\mathcal{D}_{1}$ and $e(g)=\alpha$. By applying Theorem 2 above, there exist $h, r \in \mathbb{R}\left[\left[x_{1}\right]\right]$ such that $\hat{f}=\hat{g} h+r$, as $\operatorname{Supp}(r)$ is finite. We deduce that $r$ is a polynomial and therefore $r \in \mathcal{D}_{1}$, so

$$
\widehat{f-r}=\hat{g} h \text {. }
$$

By applying of the result of Krzysztof Jan Nowak [13], Criterion of Divisibility, there exists $q \in \mathcal{D}_{2}$ such that

$$
f-r=g q .
$$

Therefore, $f$ is equivalent to $r$ modulo the ideal $I$. 


\section{THE $\left(x_{1}\right)$-ADIC TOPOLOGY OVER SOME QUASIANALYTIC LOCAL RINGS}

In the sequel, our work will be restricted just to the case of one variable. Firstly, let us recall some basic facts about the $\left(x_{1}\right)$-adic topology.

Let $m$ be an ideal of a ring $A$, the $m$-adic topology is a linear topology of the $\operatorname{ring} A$ in which the fundamental system of neighbourhoods of zero consists of the powers $m^{n}$, where $n \in \mathbb{N}$.

If the $m$-adic topology is separated (i. e. $\bigcap_{i \geq 0} m^{i}=\{0\}$ ), then this topology is metrizable. Indeed, for all $x, y \in A$, let $n$ be the largest integer such that $x-y \in m^{n}$, where $m^{0}=A$. Then we define the metric $d(x, y)=\frac{1}{2^{n}}$ when $n$ exists and 0 otherwise.

A local ring $(A, m)$ is called complete when it is complete with respect to the above metric, that is, when all its Cauchy sequences converge.

The $m$-adic completion of a ring $A$ is equal to the projective limit of the factor rings $\left(A / m^{n}\right)_{n \in \mathbb{N}}$. For example, the $\left(x_{1}\right)$-adic completion of the polynomial ring $\mathbb{R}\left[x_{1}\right]$ is the ring of formal power series $\mathbb{R}\left[\left[x_{1}\right]\right]$ up to isomorphism.

The interested reader will find more information about this in a very readable form in [1, Chapter 10]. Let $\mathcal{C}_{1} \subset \mathcal{E}_{1}$ be a quasianalytic subring of the ring $\mathcal{E}_{1}$ which contains the polynomial ring $\mathbb{R}\left[x_{1}\right]$. Assume that the ring $\mathcal{C}_{1}$ satisfies the following property, called the stability under monomial division.

Let $\hat{f} \in \hat{\mathcal{C}}_{1}$ and $\hat{f}=x_{1} \widehat{\varphi}$, where $\hat{\varphi} \in \mathbb{R}\left[\left[x_{1}\right]\right]$, then $\varphi \in \mathcal{C}_{1}$.

Remark 4. By the property of the stability under monomial division, the ring $\mathcal{C}_{1}$ is a principal ideal domain (see [2, Remark 3.1]).

Remark 5. As the polynomial ring $\mathbb{R}\left[x_{1}\right]$ is included in the ring $\mathcal{C}_{1}$ and by quasianalyticity, we may also assume that $\mathcal{C}_{1} \subset \mathbb{R}\left[\left[x_{1}\right]\right]$, and as the $\left(x_{1}\right)$-adic completion of the ring $\mathbb{R}\left[x_{1}\right]$ is isomorphic to $\mathbb{R}\left[\left[x_{1}\right]\right]$, we deduce that the $\left(x_{1}\right)$-adic completion of the ring $\mathcal{C}_{1}$ is also the ring of formal power series $\mathbb{R}\left[\left[x_{1}\right]\right]$ up to isomorphism.

Proposition 5. The polynomial ring $\mathbb{R}\left[x_{1}\right]$ is dense in the ring $\mathcal{E}_{1}$ for the $\left(x_{1}\right)$-adic topology.

Proof. Let $f$ be a smooth germ and $n \in \mathbb{N}$. We need to find a polynomial $g_{n}$ such that the germ of $f-g_{n}$ at 0 is in $\left(x_{1}^{n}\right) \mathcal{E}_{1}$. Thanks to Hadamard's Lemma 2.8 in [12], we deduce that there exists a smooth function $h_{1}$ such that $f-f(0)=x_{1} h_{1}$, and by the same way, there exists $h_{2} \in \mathcal{E}_{1}$ such that $h_{1}-h_{1}(0)=x_{1} h_{2}$.

By iterated application of the same Lemma, we can write

$$
f\left(x_{1}\right)=g_{n}\left(x_{1}\right)+x_{1}^{n} h_{n}\left(x_{1}\right),
$$

where $g_{n}\left(x_{1}\right)=f(0)+f^{\prime}(0) x_{1}+\ldots+\frac{f^{(n-1)}(0)}{(n-1) !} x_{1}^{n-1}$, and $h_{n}$ is a smooth function germ. Then the germ of $f-g_{n}$ is in $\left(x_{1}{ }^{n}\right) \mathcal{E}_{1}$.

Remark 6. Proposition 5 still holds true if we replace the ring of smooth germs $\mathcal{E}_{1}$ by the subring $\mathcal{C}_{1} \subset \mathcal{E}_{1}$ as it is closed under monomial division. 
Remark 7. From the above properties and thanks to the quasianalyticity, we deduce that the ring $\mathcal{C}_{1}$ is a metrizable separable metric space for the $\left(x_{1}\right)$-adic topology.

We end this paper by giving a criterion that allows us to test the non surjectivity of the Borel mapping over the ring $\mathcal{C}_{1}$.

Proposition 6. If the Borel mapping ${ }^{\wedge}: \mathcal{C}_{1} \rightarrow \mathbb{R}\left[\left[x_{1}\right]\right]$ is surjective, then the ring $\mathcal{C}_{1}$ is complete for the $\left(x_{1}\right)$-adic topology.

Proof. Suppose that the mapping ${ }^{\wedge}$ is surjective, and let us take $f \in \mathcal{C}_{1}$ such that $\hat{f}=x_{1}^{n} H$, where $n \in \mathbb{N}$ and $H \in \mathbb{R}\left[\left[x_{1}\right]\right]$, so by the property of the stability under monomial division, there exists $h \in \mathcal{C}_{1}$ such that $\hat{h}=H$ and by quasianalyticity, we have $f=x_{1}^{n} h$. It is clear that if $f \in\left(x_{1}^{n}\right) \mathcal{C}_{1}$ then $\hat{f} \in\left(x_{1}^{n}\right) \mathbb{R}\left[\left[x_{1}\right]\right]$, consequently, we have the equivalence $f \in\left(x_{1}^{n}\right) \mathcal{C}_{1} \Leftrightarrow \hat{f} \in\left(x_{1}^{n}\right) \mathbb{R}\left[\left[x_{1}\right]\right]$ for all $n \in \mathbb{N}$, therefore, the mapping ${ }^{\wedge}$ is a homeomorphism for the $\left(x_{1}\right)$-adic topology. As the mapping $\wedge$ is a linear homeomorphism and the ring $\mathbb{R}\left[\left[x_{1}\right]\right]$ is complete, we deduce that the ring $\mathcal{C}_{1}$ is also complete for the $\left(x_{1}\right)$-adic topology.

Remark 8. Proposition 6 still holds if we skip the assumption of stability under monomial division and by assuming just that $\mathcal{C}_{1}$ is a quasianalytic local ring which is closed under derivation.

\section{REFERENCES}

[1] Atiyah M.F., MacDonald I.G. Introduction to Commutative algebra. Addison-Wesley Publishing Company, University of Oxford, 1969.

[2] Berraho M. On a problem concerning the ring of Nash germs and the Borel mapping. AIMS Mathematics 2020, 5 (2), 923-929. doi:10.3934/math.2020063

[3] Berraho M. On definable germs of functions in expansions of the real field by power functions. Asia Mathematika 2020, 4 (2), 117-126.

[4] Bianconi R. Nondefinability results for expansions of the field of real numbers by the exponential function and by the restricted sine function. J. Symbolic Logic 1997, 62 (4), 1173-1178.

[5] Bochnak J., Coste M., Roy M.F. Géométrie algébrique réelle, Ergebnisse des Mathematik, 12. Berlin, Heidelberg, New York, Springer Verlag, 1987.

[6] Chaumat J., Chollet A.M. Division par un polynôme hyperbolique. Canad. J. Math. 2004, 56 (6), 1121-1144. doi:10.4153/CJM-2004-050-1

[7] Elkhadiri A., Sfouli H. Weierstrass division in quasianalytic local rings. Studia Math. 2008, 185 (1), 83-86.

[8] Gunning R., Rossi H. Analytic functions of several complex variables, Reprint of the 1965 original. AMS Chelsea Publishing, Providence, RI, 2009.

[9] Kaiser T. R-analytic functions. Arch. Math. Logic 2016, 55 (5-6), 605-623. doi:10.1007/s00153-016-0483-x

[10] Miller C. Expansions of the real field with power functions. Ann. Pure Appl. Logic 1994, 68 (1), 79-94.

[11] Miller C. Infinite differentiability in polynomially bounded o-minimal structures. Proc. Amer. Math. Soc. 1995, 123 (1), 2551-2555.

[12] Nestruev J. Smooth manifolds and observables. Springer, Berlin, 2002.

[13] Nowak K. On division of quasianalytic function germs. Internat. J. Math. 2013, 24 (13), 1-5. doi:10.1142/S0129167X13501115 
[14] Parusinski A., Rolin J.P. A note on the Weierstrass preparation theorem inquasianalytic local rings. Canad. Math. Bull. 2014, 57 (3), 614-620. doi:10.4153/CMB-2013-034-5

[15] Rond G. Local zero estimates and effective division in rings of algebraic power series. J. Reine Angew. Math. 2018, 2018 (737), 111-160. doi:10.1515/crelle-2015-0041

[16] Sfouli H. On a problem concerning quasianalytic local rings. Ann. Polon. Math. 2014, 111 (1), 13-20.

[17] Thilliez V. On quasianalytic local rings. Expo. Math. 2008, 26 (1), 1-23.

[18] Van den Dries L. On the Elementary Theory of Restricted Elementary Functions. J. Symbolic Logic 1988, 53 (3), 796-808.

Received 10.03.2020

Revised 08.09.2020

Беррахо М. Аеякі властивості поліноміальоно обмежених о-мінімальних продовжень дійсного поля і деяких квазіаналітичних локальних кілеиь // Карпатські матем. публ. - 2020. — Т.12, №2. — С. 483-491.

У цій роботі ми досліджуємо теорему Вейєрштрасса про подільність над кільцями гладких ростків, які можна визначити у довільному поліноміально обмеженому о-мінімальному розширенні дійсного поля, давши деякі критеріі, шо задовольняють цю теорему. Потім досліджуємо деякі топологічні властивості деяких квазіаналітичних підкілець кільця гладких ростків для $\left(x_{1}\right)$-адичної топології показуючи, що ці кільця $є$ сепарабельними метричними просторами. Також наводимо критерій їх повноти щодо $\left(x_{1}\right)$-адичної топології.

Ключові слова і фрази: теорема Вейєрштрасса про подільність, поліноміально обмежена омінімальна структура, квазіаналітичне кільце, $\left(x_{1}\right)$-адична топологія. 